\title{
Kiswahili, utandawazi na umoja wa Afrika
}

\author{
Stephen N. Kamau
}

\begin{abstract}
It is a fact that in the recent past, globalization has gradually taken shape in Africa and as such the continent has no choice but to embrace this development. In order for Africa to benefit fully from globalization, various measures must be taken. One of the key measures is the use of one indigenous African language to serve as an official and unifying language throughout the continent. We argue that the language with that potential is Kiswahili.

This paper sets out to evaluate the place of Kiswahili as a unifying language in Africa in the era of a globalizing Africa. Globalization in Africa has to do with the coming together of African nations to form a bloc that will cooperate in the social, political and economic fronts. This being the case, the need for a common African language for use across the continent cannot be ignored. The best placed African language, as this paper will demonstrate, is Kiswahili.
\end{abstract}

\section{UTANGULIZI.}

Karne ya ishirini na moja kwa kweli ni muhimu katika historia ya ulimwengu mzima. Hii ni karne ambayo imeshuhudia kuingia kwa utandawazi kwa kiwango cha juu sana ikilinganishwa na karne zingine zilizopita. Kwa hakika, utandawazi si tukio jipya ulimwenguni bali umekuwepo kwa karne nyingi japo kwa viwango vya chini ikilinganishwa na karne hii ya ishirini na moja. Kuingia kwa utandawazi kumeleta maingiliano ya hali ya juu ya mataifa ulimwenguni. Kama asemavyo Ryanga (2002), utandawazi umeleta maingiliano kati ya mataifa yaliyoendelea na yale ambayo yanaendelea na pia kati ya mataifa makubwa na yale madogo. Hapa Afrika, kama ilivyo katika mabara mengine ulimwenguni, utandawazi tayari umeingia na kuanza kushika mizizi kwa dhati. Hali ikiwa hivi, lazima bara hili litafute namna ya kunufaika kutokana na utandawazi. Kwa ufupi, makala haya yanalenga kulijadili swala la Kiswahili na utandawazi na jinsi maswala haya mawili yanavyoweza kuchangia katika kuleta umoja wa Afrika.

\subsection{UTANDAWAZI NI NINI?}

Utandawazi kulingana na Sullivan (1994) ni ule usambaaji wa maswala mbali mbali kama vile biashara, mawasiliano, utamanduni n.k kutoka kwa kiwango cha kitaifa hadi katika kiwango cha kimataifa. Akifafanua zaidi, yeye anasema kuwa utandawazi ni ule usambaaji wa maswala kama haya nje ya mipaka ya kitaifa kwa kipindi cha miaka ishirini (20) iliyopita.

Naye Mwaro (2001), asema kuwa utandawazi una maana ya mtando ambao huleta utengamano wa kimataifa na vile vile ongezeko la haraka la ubadilishanaji maarifa katika eneo pana. Akiongezea, yeye anafafanua kuwa utandawazi ni nguvu zinazougeuza ulimwengu na kuufanya kama kijiji haswa kupitia upanuzi wa teknolojia ya habari na mawasiliano (TEKNOHAMA). 
Ryanga (2002) aueleza utandawazi kuwa utambuzi wa mataifa ya ulimwengu kuwa lazima yafanye kazi pamoja, yashirikiane kibiashara, kiteknolojia, na katika kufanya uwamuzi unaohusu maswala yanayoathiri ulimwengu.

Kwa ufupi, utandawazi unahusu ushirikiano wa kimataifa katika maswala mbali mbali yanayohusiana na siasa, biashara, uchumi, michezo n.k. Kwa sasa ushirikiano huu unawezeshwa na pia kurahisishwa na teknolojia ya habari na mawasiliano (TEKNOHAMA) ambayo kwa kweli imeuunganisha ulimwengu mzima kimawasiliano.

\subsection{UTANDAWAZI NA KISWAHILI.}

Hali ilivyo kwa sasa ni kuwa utandawazi unalifikia bara la Afrika kupitia lugha za kigeni kama vile Kiingereza na Kifaransa. Hali hii yafaa kutupiliwa mbali iwapo bara letu linatarajiwa kunufaika kikamilifu kutokana na utandawazi. Kwa vile utandawazi huhusika na uunganishaji wa mataifa mbali mbali, basi Afrika yahitaji lugha moja yenye asili ya Kiafrika kama lugha yakusambaza utandawazi.

Kwa sasa mfano mzuri wa lugha kama hii ni lugha ya Kiswahili. Lugha hii ndiyo lugha kutoka barani Afrika ambayo ina matumizi mapana zaidi kuliko lugha nyingine hapa barani na kwingineko. Basi utandawazi unaweza kusambazwa vizuri zaidi kupitia Kiswahili. Katika kufanya hivi, lugha hii pia itakuwa imefaulu kwa kiasi kikubwa katika kuleta umoja barani Afrika. Bila shaka, bali na umoja wa Afrika, bara hili pia litapata manufaa mengine mengi ikiwa tu utandawazi utayafikia mataifa ya Afrika yakiwa yameungana na kuwa jimbo moja.

Kulingana na Amatubi (2002), utandawazi husambazwa kupitia itikadi, teknolojia, na lugha. Hata hivyo, anafafanua zaidi kuwa lugha ndicho huwa chombo muhimu zaidi katika usambazaji wa utandawazi. Hapa Afrika, kama tayari ilivyofafanuliwa, Kiswahili ndiyo lugha mwafaka ya utandawazi na ambayo inaweza kuhakikisha kuwa bara hili limeukabili utandawazi likiwa kama jimbo moja.

Ijapokuwa Kiswahili kimetiliwa shaka na wengi kama lugha ambayo inaweza kutumika katika kupitisha teknolojia mpya zinazoibuka, utafiti uliofanywa umedhirisha kuwa lugha zote ulimwenguni zina uwezo wa kuzimudu teknolojia hizi mpya kwa njia ya uundaji wa msamiati mpya.Mazrui na Mazrui (1995:25). Wanaedelea kufafanua kuwa tayari Kiswahili kimejidhihirisha kuwa lugha ambayo inaweza kuzimudu teknolojia mpya katika nyanja mbali mbali. Kwa mfano, katika nyanja ya sayansi na teknolojia, tayari kuna kamusi za fiskia, biolojia na vile vile msamiati wa uundaji wa magari. Kwa sasa, Kiswahili kinatumika katika mitambo ya tarakilishi. Kwa hakika, mifano hii yote ni dhihirisho tosha kuwa Kiswahili ni lugha ambayo kwa kweli inaweza kuyakabili karibu maswala yote ya kiteknolojia yanayotokea ulimwenguni. 


\subsection{KUINGIA KWA KISWAHILI KATIKA RAMANI YA UTANDAWAZI.}

Lugha ya Kiswahili kwa kweli ina historia ndefu kama lugha ya utandawazi. Hii ni kwa sababu lugha hii ilianza kutumika kama lugha ya kimataifa kitambo sana. Kwa hakika lugha ya Kiswahili iliwekwa katika ramani ya utandawazi kupitia biashara, haswa ile ya utumwa, ambayo ilikuwepo kati ya miji ya pwani ya Afrika mashariki kwa upande mmoja na mataifa ya Uarabuni, Asia na Uropa kwa upande ule mwingine. Biashara hii iliweza kukisambaza Kiswahili nje ya mipaka ya pwani ya Afrika Mashariki na pia kukieneza katika maeneo ya bara hapa Afrika.

Bali na biashara, wamishenari nao walipofika walisaidia sana katika kufanikisha utandawazi wa Kiswahili. Wao walihakikisha kuwa lugha hii imeyafikia mataifa ya Kimagharibi kupitia uchapishaji wa vitabu kama vile kamusi na vitabu vya isimu ya Kiswahili. Waliotia fora zaidi ni wamishenari wa Kijerumani ambao walichangia sana katika uchapishaji na utafiti wa lugha ya Kiswahili.

Vita vya kwanza na vya pili vya dunia navyo vilitoa mchango wa kipekee katika kusambaza lugha ya Kiswahili katika maeneo mapya ulimwenguni. Lugha hii ilitumika na wanajeshi kama lugha ya mawasiliano hapa Afrika Mashariki na kati. Wanajeshi hawa walipofika katika sehemu zingine ulimwenguni waliweza kuifikisha lugha hii huko.

Jambo lingine ambalo limesaidia katika kukifanya Kiswahili lugha ya utandawazi ni kule kuanzishwa kwa vituo vya masomo ya Kiafrika katika vyuo vikuu vingi kule Uingereza, Marekani na Ujerumani. Katika vituo hivi, lugha ya Kiswahili huwa ni kipengele kimoja muhimu katika programu zao za kimasomo. Lugha hii huwa muhimu kwa vyuo hivi haswa vinapofanya utafiti barani Afrika.

Shughuli za utangazaji pia zimechangia katika kukisambaza Kiswahili ulimwenguni kote na hivyo kukifanya kuwa lugha ya utandawazi. Idhaa maarufu za utangazaji duniani kama vile B.B.C, V.O.A, Dutche Welle, Radio China, Radio Moscow n.k huwa na vipindi vya matangazo kwa Kiswahili. Idhaa hizi basi huchangia katika kuikuza lugha ya Kiswahili kama lugha ya kimataifa.

Kupitia njia kama hizi, lugha ya Kiswahili imejitokeza kuwa lugha ya kipekee barani Afrika ambayo imeyapata matumizi ya kimataifa na hivyo kuwa lugha ya utandawazi. Hata hivyo, kunazo sababu maalum ambazo kwa kweli zinakifanya Kiswahili kupendekezwa na wengi kuwa lugha ya utandawazi barani Afrika. Sababu hizi zimejadiliwa katika sehemu inayofuata.

\subsection{SABABU ZA KISWAHILI KUWA LUGHA YA UTANDAWAZI BARANI AFRIKA.}

Kiswahili kwa kweli ni lugha ambayo imetetewa na wataalam wengi wa lugha kuwa kama lugha ya utandawazi barani Afrika. Wataalam hawa pia wanapendekeza kuwa Kiswahili chafaa kuwa lugha pekee ya matumizi mapana barani Afrika iwapo bara hili lingetaka kuwa na maendeleo dhabiti bali na kuwa na umoja. Kati ya sababu zilizotolewa na wataalam hawa ni kama vile:- 
a) Kiswahili ndiyo lugha ambayo ina ukubalifu wa kimataifa kuliko lugha nyingine yoyote barani Afrika kwani ni lugha yenye utaifa mwingi. Hii ni kumanisha kuwa lugha hii haina tisho la kisiasa au kiuchumi kutokana na ukweli kuwa wenyeji wake hawana uwezo wa kisiasa au kiuchumi. Kwa sababu hii Kiswahili kinachukuliwa kuwa kama lugha ya kila mtu. (Nyaigoti Chacha akinukuliwa na Mwaro 2002).

b) Lugha ya Kiswahili ndiyo lugha ya Kiafrika ambayo ina matumizi mapana zaidi hapa Afrika. Pia, lugha hii ndiyo iliyosambaa zaidi katika kiwango cha kimataifa katika maeneo kama vile Marekani na Uropa. Amatubi (2002) anasema kuwa Kiswahili ni lugha mwafaka sana ya utandawazi barani Afrika kwani tayari ni lugha ya kimataifa. Hapa Afrika, lugha hii tayari inazungumzwa katika nchi kama vile Tanzania, Kenya na Uganda. Pia inazungumzwa katika sehemu fulani za Rwanda, Burundi, Malawi, Musumbiji, Somalia na nchi ya kidemokrasia ya Congo.

c) Kiswahili pia kimekuwa lugha ya kutambulika ulimwenguni kama lugha ya utangazaji na uchapishaji. Kama ilivyofafanuliwa hapo awali (tazama 2.1), lugha hii inatumiwa katika utangazaji na idhaa nyingi za utangazaji hapa Afrika na kwingineko duniani.

d) Pia kama asemavyo Mwaro (2002), Kiswahili ni lugha ambayo ni rahisi kujifunza na kuitumia kutokana na kiwango chake cha juu cha usimilisho. Kwa mfano, kama aelezavyo Amatubi (2002), wageni wengi kutoka nchi za magharibi hutokea kuwa wazungumzaji hodari wa Kiswahili katika mwaka wao wa kwanza nchini Kenya kama vile wakoloni na wamishenari.

e) Kiswahili pia ni lugha ambayo inaweza kuzimudu teknolojia mpya zinazoibuka kwa urahisi kupitia kwa uundaji wa msamiati mpya (Mazrui na Mazrui 1995).

\subsection{UTANDAWAZISHWAJI WA KISWAHILI BARANI AFRIKA.}

Ikiwa lugha ya Kiswahili ndiyo inayokubalika zaidi kama lugha ya utandawazi barani Afrika, basi hatua kadhaa zafaa kuchukuliwa ili kuhakikisha kuwa lugha hii imefanikiwa katika kulitekeleza jukumu hili.

Kwanza kabisa, ili utandawazi wa Kiswahili uwepo barani Afrika, lazima utegemezi wa lugha za kigeni ukomeshwe kabisa kote barani. Hali iliyopo kwa sasa ni kuwa mataifa mengi ya Kiafrika hutegemea lugha za kimagharibi haswa zile za wakoloni waliokuwa wakizitawala. Lugha za kimagharibi ambazo hutegemewa sana na mataifa mengi hapa barani Afrika ni Kiingereza, Kifaransa na Kireno. Pia lugha ya Kiarabu nayo ni lugha nyingine ya kigeni ambayo hutegemewa sana na Waafrika japo si ya asili ya kimagharibi. Utegemezi wa lugha hizi za kigeni kwa kweli huzinyima lugha za Kiafrika kama vile Kiswahili nafasi ya kukua na kutegemewa kama lugha za utandawazi barani Afrika. Zaidi lugha hizi huwa hazieleweki na idadi kubwa ya Waafrika na kwa hivyo wao hawanufaiki kikamilifu kutokana na utandawazi. 
Kulingana na Mwaro (2002), tayari mataifa mengine duniani yameanza kwa dhati juhudi za kutandawazisha lugha zao za kiasili ili zinufaike kikamilifu kutokana na utandawazi. Mataifa haya ni kama vile Japani, Israeli, Ujerumani, Uchina na Korea. Bara la Afrika lafaa kuiga mataifa kama haya na kuanza kutandawazisha lugha zake za kiasili ili kukabiliana na utandawazi kwa lugha zinafahamika vyema na Waafrika wengi. Kama anavyofafanua zaidi, Bara la Afrika litaingia katika utandawazi kupitia juhudi pana za kujitegemea na kuondoa umelea wa kutegemea lugha za kigeni kimsamiati. (Mwaro 2002:300).

Pili, lugha ambayo inanuiwa kutandawazishwa barani Afrika lazima ikuzwe kimsamiati ili iweze kukabiliana na teknolojia na maswala mengine mapya yanayoletwa na utandawazi. Lugha ya Kiswahili tayari imejitokeza kuwa lugha ambayo inaweza kupanuliwa kimsamiati kwa urahisi na kwa hivyo ina uwezo mkubwa wa kuwa lugha ya utandawazi barani Afrika.

Kufikia sasa lugha hii imeundiwa msamiati mwingi wa kitaaluma kuliko lugha nyingine ile hapa Afrika.(tazama 2.0). Pia, kwa sasa lugha ya Kiswahili imepiga hatua kubwa ya kimaendeleo kwa kuwa lugha ya kwanza ya Kiafrika kuwahi kutumika kama lugha ya matumizi katika tarakilishi. Juhudi hizi zinatekelezwa na kampuni ya Microsoft East Africa. Shughuli hii bado inaendelea ikiwa katika awamu yake ya pili. Awamu ya kwanza ya mradi huu tayari imekamilika na istilahi elfu tatu (3 000) za Kiswahili kuundwa tayari kwa matumizi katika tarakilishi.

La muhimu hapa ni kuwa lugha ya Kiswahili itaweza kutumika katika tarakilishi ambacho huwa chombo muhimu katika utandawazi. Kama asemavyo Khayesi (2002), TEKNOHAMA ni kiungo muhimu sana cha utandawazi. Kwa hivyo, matumizi ya Kiswahili katika tarakilishi yatakiingiza kikamilifu katika TEKNOHAMA. Ikiwa hivi basi, lugha ya Kiswahili ndiyo lugha ya Kiafrika ambayo kwa kweli ina nafasi bora zaidi ya kuwa lugha ya utandawazi barani.

Tatu, Umoja wa Afrika wafaa kuwa katika mstari wa mbele katika kupigania matumizi ya Kiswahili kama lugha ya utandawazi barani Afrika. Umoja wa Afrika wapaswa kutazama upya juu ya sera yake ya matumizi ya lugha za kigeni katika vikao vyake iwapo una nia ya kuzikuza lugha za Kiafrika kama vile Kiswahili kuwa lugha ya matumizi mapana barani. Kwa njia hii utasaidia katika kuleta umoja wa Afrika.

Hata ingawa lugha ya Kiswahili tayari imechaguliwa kama lugha mojawapo ya matumizi katika vikao vya Umoja wa Afrika, bado washiriki karibu wote hupendelea kutumia lugha za kimagharibi kwenye vikao hivi. Umoja wa Afrika unapaswa kutumia lugha ya Kiswahili pekee katika vikao vyake ikiwa lugha hii inatarajiwa kuwa lugha ya utandawazi hapa barani Afrika. Jambo hili haliwezi likatokea kwa ghafla bali, kama asemavyo Ryanga (2002), linahitaji muda wa kama miaka mitano itakayoshuhudia shughuli mbali mbali za kukisambaza Kiswahili kote barani. Anapendekeza kuundwa kwa taasisi ya Kiswahili ya Afrika nzima ambayo itashughulika na maswala yote ya utandawazishaji wa Kiswahili kote barani Afrika. 


\subsection{UTANDAWAZI WA KISWAHILI NA UMOJA WA AFRIKA.}

Kufikia hapa, makala haya yamekuwa yakijaribu kutetea matumizi ya Kiswahili kama lugha ya utandawazi barani Afrika. Kwa ufupi, kukifanya Kiswahili lugha ya utandawazi barani Afrika kunamaanisha kuwa lugha hii itakuwa ikitumika na mataifa yote barani Afrika. Hili bila shaka ni jambo lililo ngumu kutekeleza lakini si kusema kuwa haliwezekani kabisa.

Kulingana na Ryanga (2002), utandawazi hukabiliwa na mataifa mbali mbali ulimwenguni kwa namna tofauti tofauti. Mataifa yenye nguvu hukabiliana na utandawazi kibinafsi huku mataifa mengine yakija pamoja na kuunda jimbo kwa minajili ya kuukabili utandawazi. Mifano ya majimbo kama haya ni kama vile Group 77 na European Union. Kwa hivyo, yeye anapendekeza kuwa mataifa ya Afrika yafaa kukabiliana na utandawazi yakiwa kama jimbo moja chini ya Umoja wa Afrika. Kwa njia hii, bara la Afrika litaweza kufaidika kibiashara, kiteknolojia na kisiasa kutokana na utandawazi.

Akilifafanua zaidi swala hili, Ryanga asema kuwa ili bara la Afrika liweze kushiriki kikamilifu katika utandawazi likiwa kama jimbo, lazima basi lisisitize swala la matumizi ya lugha moja ambayo itatumika barani kote. Iwapo lugha ya Kiswahili itafanywa kuwa lugha ya utandawazi barani Afrika, manufaa mengi bila shaka yatapatikana. Kati ya manufaa haya ni kule kupatikana kwa umoja wa Afrika. Hii inamaanisha kuwa mataifa ya Afrika yatakuwa yameunganishwa na kuwa kama jimbo moja kimawasiliano. Lazima ikumbukwe kuwa mawasiliano ndiyo ambayo kwa kweli hutegemewa kwa kiwango cha juu katika harakati zote za utandawazi.

Bara la Afrika lina mataifa hamsini na nne (54) na bila shaka lugha chungu nzima za kiasili bali na zile za kigeni. Hali hii imekuwepo kwa muda mrefu na kwa hivyo si jambo rahisi vile kuanzisha matumizi ya lugha moja kote barani. Upinzani na ushindani mwingi bila shaka utazuka. Kwa upande mmoja, makabila haswa yale makubwa barani Afrika, yatang'ang'ania lugha zao zichaguliwe kwa shughuli hii. Mifano ya lugha zingine za Kiafrika ambazo zinaweza kukipa Kiswahili upinzani ni kama vile Kizulu cha Afrika Kusini na Kiyoruba, Kihausa na Kiibo kutoka Nigeria. Hata hivyo, lugha hizi bado hazijapata umaarufu wa kimataifa kama ule wa Kiswahili.

Kwa upande ule mwingine, upinzani mwingine utatoka kwa lugha kuu za kigeni ambazo zinazungumzwa hapa Afrika kama lugha za matumizi mapana. Lugha hizi ni kama vile Kiingereza, Kifaransa, Kireno na Kiarabu. Lugha hizi huchukuliwa kama lugha za kifahari na Waafrika na kwa hivyo itakuwa jambo ngumu kuondoa kasuba hii. Zaidi, mataifa ambako lugha hizi zimetoka nayo hupigania sana kuzikuza hapa barani kama mbinu moja ya kuendeleza ukoloni mamboleo.

Hata hivyo, lazima mataifa yote barani Afrika yajaribu kwa vyovyote vile kutupilia mbali upinzani na ushindani kama huu kwa kuuangazia kwanza manufaa yanayoweza kupatikana iwapo lugha moja ya Kiafrika itatumika kote barani Afrika. 
Faida kuu hapa ni umoja wa Afrika ambao ni ufunguo wa maendeleo mengi na ya haraka.

Hapa barani Afrika, nchi ya Tanzania ni kielelezo kizuri cha kudhibitisha jinsi umoja wa kitaifa unavyoweza kupatikana kupitia matumizi ya lugha moja ya Kiafrika katika shughuli zote za nchi. Nchi ya Tanzania ina makabila na lugha zaidi ya mia moja lakini makabila haya yote yameunganishwa pamoja na matumizi ya lugha moja yaani Kiswahili. Umoja wa Afrika basi unapaswa kuazima mengi kutoka kwa nchi ya Tanzania iwapo utafikia uwamuzi wa kikitumia Kiswahili kama lugha ya utandawazi barani Afrika. Nchi hii inazo taasisi zilizokomaa za kukuza Kiswahili kama vile T.U.K.I ambazo zingesaidia sana katika kuunda taasisi ya uchunguzi wa Kiswahili ya Afrika nzima.

Tayari bara la Afrika limeanza kushirikiana katika nyanja zingine kama vile biashara na siasa. Katika biashara, ushirikiano unawezeshwa na mikataba ya kibiashara kama ule wa COMESA. Vile vile, mataifa haya hushirikiana katika siasa haswa katika kule kudumisha amani katika mataifa yaliyokumbwa na vita. Hapa utayapata mataifa mbali mbali yakituma vikosi vya kuweka amani katika mataifa yenye michafuko ya kivita. La kuvuja moyo ni kuona kuwa ushirikiano mwingi wa aina hii hutekelezwa kupitia lugha za kigeni.

Swali kuu hapa ni kuwa, iwapo mataifa ya Afrika hushirikiana katika nyanja kama hizi kwa nini basi yasishirikiane pia katika nyanja ya lugha? Labda ushirikiano katika kiwango cha lugha ndio uliosalia katika kupata umoja wa Afrika pindi bara hili linapozidi kukabiliana na utandawazi.

\subsection{HITIMISHO.}

Kutokana na mjadala uliozuka kwenye makala haya, ni wazi kuwa utandawazi tayari umeingia barani Afrika na basi bara hili halina lingine bali kuukaribisha. Jambo lingine lililojitokeza ni kuwa ili kunufaika kikamilifu kutokana na utandawazi, lazima bara hili litumie lugha moja ya Kiafrika kama lugha ya matumizi mapana. Lugha yenye nafasi nzuri ya kufanya haya na iliyopendekezwa hapa ni Kiswahili. Matumizi ya Kiswahili kama lugha rasmi ya mawasiliano barani yataleta umoja wa Afrika na hatimaye maendeleo dhabiti.

Kama ilivyofafanuliwa, hili si jambo rahisi na lina changamoto zake. Kati ya changamoto zitakazokumba shughuli hii ni kule kuwepo kwa lugha ambazo wazungumzaji wake wangependelea zichaguliwe badala ya Kiswahili. Pia, lugha za kigeni zinazotumika kwa sasa barani Afrika zitapigania kubakia katika nafasi zake za sasa. Aidha, hii ni shughuli ambayo inaweza kuwa na gharama kubwa bali na kule kuchukuwa muda mrefu.

Hata ingawa kuna changamoto kama hizi, faida zinazoweza kupatikana iwapo Afrika itaungana kupitia lugha ya Kiswahili ni nyingi kiasi cha kuendelea kupuuzwa. Basi Waafrika wote wafaa kusisahau tofauti zao zote za lugha na kuanza kukitukuza Kiswahili kama lugha rasmi ya mawasiliano barani Afrika. Penye nia pana njia! 


\section{MAREJEREO.}

Amatubi, M.N. (2002) 'Globalization and the mainstreaming of Kiswahili research in the $21^{\text {st }}$ Century ' katika Utafiti wa Kiswahili, Maseno, Maseno University. uk. $43-48$.

Kamau, S.N. (2005) Matumizi ya Kiswahili katika Tarakilishi, Masters Thesis (Unpublished): Kenyatta University.

Katembo,I.B. (2005) "Kiswahili, the Global Economy and the African American". Seminar paper presented at the International Symposium on Kiswahili language and Globalization. University of Dar es Salaam,Tanzania , July $4-7,2005$

Khayesi, M.K. (2002) 'Kiswahili and Information Technology (I.T) : Challenges in Public University Libraries in Kenya' katika Kiswahili Juzuu 65 Dar- es Salaam, T.U.K.I. uk. $24-31$.

Mazrui na Mazrui. (1995) Swahili State and Society, Nairobi,East African Educational Publishers.

Mbaabu, I. (1989) ‘Ukubalifu wa Msamiati: Mifano kutoka Kenya' katika Makala ya Semina ya Kimatifa ya usanifishaji wa Istilahi za Kiswahili, Dar-es Salaam, T.U.K.I. uk. $23-28$.

Mwaro, A.G. (2002) ‘Mchango wa A.S.Nabhany katika Utafiti na Utandaridhi wa Istilahi za Kiswahili' katika Utafiti wa Kiswahili, Maseno, Maseno University. Uk $49-56$.

Ryanga, S. (2002) 'The African Union in the Wake of Globalization: The Forgotten Language Dimension' katika Kiswahili Juzuu 65 Dar-es-Salaam, T.U.K.I uk. $1-15$.

Sullivan, T. (1994) Key Concepts in Communication and Cultural Studies, Canada, Routledge Press. 